\title{
Editorial
}

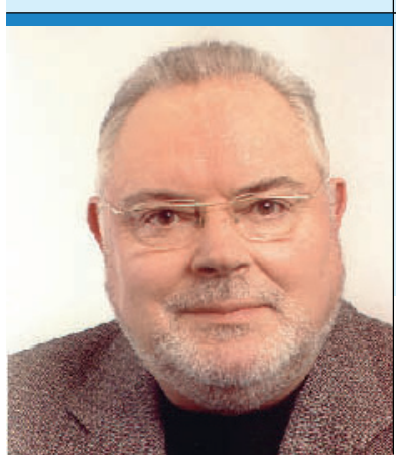

"Um die Wissenschaftskultur zu erhalten, werden die

Hochschulen in Zukunft genauer hinschauen müssen."

Dr. med. Dieter Leithäuser,

Chefredakteur und niedergelassener HNO-Arzt, Warburg

\section{Noch ist die Wissenschaft nicht verloren}

D er Schaden, den prominente Plagiatoren der jüngsten Zeit wie zu Guttenberg, Koch-Mehrin und Stoiber-Tochter Veronica Saß angerichtet haben, geht über private Belange weit hinaus. Zwar befinden sich die Herrschaften durchaus in der guten Gesellschaft berühmter historischer Persönlichkeiten, und die Liste historisch bedeutender Plagiate ist ziemlich lang. So hat zum Beispiel William Shakespeare keine einzige Figur seiner Dramen selbst erfunden, und doch wird niemand behaupten, dass er ein Betrüger wäre. Denn aus den farblosen, hölzernen Figuren seiner Vorlagen schuf er unverwechselbare Persönlichkeiten mit psychologischem Tiefgang. Thomas Mann etwa bekannte sich offen dazu, abgeschrieben zu haben. In seinen „Buddenbrooks“ stirbt am Ende der junge Hanno an Typhus. Dafür bediente sich der Schriftsteller aus einem medizinischen Fachbuch. Dadurch, dass er die nüchterne Beschreibung des Krankheitsverlaufs im Kontext seines Romans wiedergibt, wirkt diese jedoch völlig anders - nämlich zutiefst schockierend.

Viele große Schreiber haben aus dem Abkupfern erst gar keinen Hehl gemacht. Der Dichter Heinrich Heine etwa bezeichnete Plagiatsvorwürfe als „töricht“. Johann Wolfgang Goethe schrieb: „Die sämtlichen Narrheiten von Plagiaten und Halbentwendungen erscheinen mir läppisch." $\mathrm{Zu}$ seinen Lebzeiten gab es noch kein Urheberrecht wie heute.

Dennoch: Die bekannten Plagiatoren der Neuzeit haben erheblichen Schaden angerichtet. Für uns, die ihren Doktortitel „ehrlich“, ohne Computer-, Wiki- pedia- und Googlehilfe erworben haben, bricht zwar keine Welt zusammen. Die Sorge gilt aber dem Wissenschaftsbetrieb, dessen Ruf nicht nur von einzelnen Betrügern, sondern darüber hinaus auch noch durch die hohe Politik beschädigt wird. Es sei an die Worte von Frau Merkel erinnert, sie brauche einen Minister und keinen wissenschaftlichen Mitarbeiter. Dass die Wissenschaft von der Politik ungestraft verhöhnt wird, ist alarmierend.

Natürlich ist durch die Plagiatsaffäre auch das Ansehen der Doktorväter deutlich ramponiert. Konnten oder wollten die sonst so souveränen Herren, einschließlich der obligaten Berichterstatter, nicht so genau hinschauen? Wurden akademische Benotungen wie „Summa cum laude“ mit Abzählreimen festgelegt? Meine Doktorarbeit erhielt 1973 nur ein schlichtes "Cum laude“" weil sie nicht experimentellen, sondern nur klinischen Ursprungs war. Trotz alledem bleibt festzuhalten: Die Hochschulen haben gezeigt, dass sie die Plagiatoren bestrafen können.

Fazit: Die Universitäten werden in Zukunft genauer hinschauen müssen und Titel weniger blauäugig vergeben - ein kleiner Schritt für die Wissenschaft, aber ein großer für unser Bemühen die Wissenschaftskultur zu erhalten.

Ihr
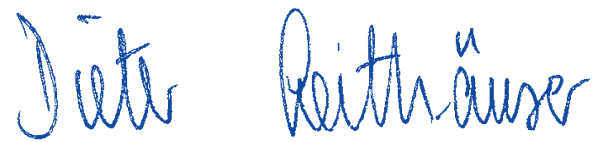